\section{Stress im Spital}

\author{
E. Taverna
}

Die Krankenschwestern hiessen Hartwiga und Norberta. Die eine war Chefhebamme, die andere kommandierte den Operationssaal, und auf der Hierarchiestufe des Spitals rangierten sie nahe beim Chef, sehr nahe. Sie drillten die Unterassistenten und neuen Assistenten und ihre Anordnungen waren ausnahmslos zu befolgen, selbst dann, wenn sie befanden, dass Chirurgenhosen den Frauen zu verbieten seien. Wer es mit ihnen verdarb, hatte schlechte Chancen. Nur das Röntgen und das Labor waren weltlich bestimmt.

Die Schwestern lebten, ausserhalb ihrer überlangen Arbeitszeit, in einem für uns unerreichbaren Bezirk. Dort herrschte der Mann am Kreuz, und in seinem Reich war das Spital nur eine irdische Provinz. Selten blieb ein Patient kürzer als zwei Wochen, oft genug dauerte der Aufenthalt einen Monat, und Wöchnerinnen blieben prinzipiell zwölf Tage, weil Landfrauen auch einmal Ferien und die übrigen von ihren Männern eine Schonung bräuchten. Doch diese Zeit der Menzinger, Baldegger, Ingenbohl und anderer Mutterhäuser ist bei uns unwiederbringlich abgelaufen.

\section{Die Studie}

Die Spitäler von Wetzikon, Lugano, Nyon, Morges, Martigny, die Psychiatrischen Universitätskliniken Genf und Zürich sowie das Kantonsspital Schaffhausen haben sich an der Pilotstudie beteiligt. In der Schweiz gehören 18 Betriebe und Verbände zum Netzwerk "Gesundheitsfördernder Krankenhäuser", zu einem WHO-Verbund mit weltweit über 500 Spitälern. Das Projekt arbeitete mit den Methoden der Fokusgruppe, das heisst mit moderierten, bis zweistündigen Gesprächen mit jeweils 8-12 der total 114 Teilnehmer. Vertreten waren 23 verschiedene Berufe, darunter 63 Krankenschwestern. Passagen der Tonbandaufzeichnungen sind in der Studie «Das Spitalpersonal bewältigt den Stress" ausführlich zitiert. Der Begriff Stress wurde durch folgende Aspekte definiert: Nicht-Beherrschung der Situation durch übliche Mittel; Unvorhersehbarkeit; subjektiver Eindruck, ohne adäquate Mittel dazustehen; Forderung, neue Antworten und Neuerungen zu finden; Gefühl des fehlenden Verständnisses. Die Ergebnisse gliedert der 55seitige Bericht in 10 Kapitel. Sie beschreiben die vorhandenen Strukturen, Direktion und Behörden, Ärzteschaft und Pflegepersonal, Innovationen, Bastelwerke und Änderungen, berufliche Verpflichtungen, Patienten als Stressfaktoren, Unsicherheit, Machtlosigkeit, Berufsbild, Qualität und Sicherheit. Analysen der Strategien im Umgang mit Stress und der Auswirkungen auf Beruf und Privatleben beenden die Gespräche. Häufige Klagen betreffen den Per- sonalmangel, die Hypermobilität, die Arbeitszeiten und Überstunden, den Papierkram, aber auch die Anwesenheit von immer mehr Stagiaires, Lernschwestern und Aushilfspersonal. Häufig sind auch die Klagen über enge und übernutzte Räume, die ein konzentriertes Arbeiten verhindern. Die fehlende Betreuung gefährdet das Ausbildungsziel und führt zu immer mehr Aussteigern. Als problematisch werden oft auch die Wechselbeziehungen zwischen Ärzten und Schwestern empfunden. Vor allem Verständigungsprobleme machen zu schaffen. Energieverschleiss und Zeitverschleuderung sind die Folge: "Je weiter es geht, desto mehr hat man den Eindruck, sich nicht mehr zu verstehen und auf parallelen Spuren zu gehen, die sich niemals treffen werden.» "Und der Arzt, das ist seine Art, den anderen aufzuwerten, indem er ihn noch schuldbewusster macht, sagt: Ach, das ist unmöglich, aber immerhin, Sie müssen es ja wissen, wie es geht ...» Ein Konfliktort erster Ordnung scheint der Operationssaal zu sein, wo überladene Programme, schlechte Organisation und fehlendes Personal Spannungen und Machtkämpfe erzeugen. Angeprangert werden Verspätungen, fehlende Erfahrung, vage Anweisungen und vor allem die fehlende Betreuung junger Ärztinnen und Ärzte, die in Umkehrung der sonst geltenden Hierarchie durch die Schwestern angeleitet werden müssen: «Ja, sie stehen unter extremem Stress und ich verbrauche mehr Energie, um sie zu überwachen, zu unterstützen, ihnen zu helfen, als mich um die Patienten zu kümmern.» Weitere Bemerkungen betreffen die mangelnde Anerkennung und das schiere Unwissen über die Kompetenzen des Pflegepersonals. Nicht nur die Ärzte werden kritisiert, für deren Situation es trotz Vorbehalten auch viel Verständnis gibt. Verwaltung, Politiker und Patienten tragen auch zum schwierigen Alltag bei. Und natürlich sind da auch die berufstypischen Probleme: die Angst vor Fehlern, die Ohnmacht gegenüber Krankheit und Tod, die Notfallsituationen und der dauernde Druck.

\section{Auswege gesucht}

Der professionelle Alltag spiegelt den gesellschaftlichen Wandel mit seinen tiefgreifenden Strukturänderungen. Wie kann da die öffentliche Spitalwelt eine heile Insel sein? Sie ist überspezialisiert, überbürokratisiert, unter enormem Zeitdruck und immer noch von Lohnungleichheiten geprägt. Aber sie ist auch hocheffizient, und niemand würde im Ernst freiwillig auf die vielen Dienstleistungen verzichten. Versäumnisse weisen alle auf: die Gesundheitspolitiker, die sich der angeblichen Defizitwirtschaft entledigen möchten, die medizinischen Chefs, die der zunehmenden Überlastung ihres eigenen Nachwuchses tatenlos zugeschaut haben, und die Pflegeschulen, die nicht immer vor der Versuchung gefeit sind, mit alten Feindbildern neue Hierarchien aufzubauen. Es hat den Bleistiftstreik und Protestumzüge gebraucht, um längst bekannte Mängel publik zu machen. Die Rezepte und Expertenvorschläge sind immer die glei- 
chen: Diskussionsforen, Fragebogenaktionen, Supervisionen, mehr Beratungsstellen, neue Studien, freiwillige medizinische Untersuchungen und Stresskonsultationen. Als ob mit Organisation und Nett-seinMiteinander allein die Probleme ausgeräumt wären.

Die Studie wiederholt, was ein Buch 1998 als «Ende der Gemütlichkeit» beschrieben hat. Das «strukturelle Unglück und mentale Leid in der Schweiz», wie der Untertitel lautet, wurde durch eine Sammlung von Porträts quer durch das Land beschrieben. Gemeint war mit Gemütlichkeit das Ende von dem, was «heimisch, vertraut, geschützt, beständig, warm und behaglich scheint, was auch mit Überschaubarkeit und
Stabilität beschrieben werden könnte». Die Lebensgeschichte «Stress im Spital. Die Leiden einer Krankenschwester" liest sich wie eine Zusammenfassung der vorliegenden Studie. Sie ist eine von 30 Erzählungen, die dem gesellschaftlichen Umbruch und dem tiefen, diffusen Unbehagen ein Gesicht geben. Weil die Fallstudien das Spital nicht isoliert betrachten, sind sie hochaktuell und lesenswert.

- Undritz N. Einblick in die Stresslandschaft der Krankenhäuser. Suhr: N. Undritz; 2001. www.undritz.ch.

- Honegger C, Rychner M (Hrsg.). Das Ende der Gemütlichkeit. Zürich: Limmat; 1998. 